General Mathematics Vol. 27, No. 2 (2019), 71-83

DOI:10.2478/gm-2019-0016

Sciendo

\title{
Improved error estimate and applications of the complete quartic spline ${ }^{1}$
}

\author{
Alexandru Mihai Bica, Diana Curilă, Zoltan Satmari
}

\begin{abstract}
In this paper an improved error bound is obtained for the complete quartic spline with deficiency 2 , in the less smooth class of continuous functions. In the case of Lipschitzian functions, the obtained estimate improves the constant from Theorem 3, in J. Approx. Theory 58 (1989) 58-67. Some applications of the complete quartic spline in the numerical integration and in the construction of an iterative numerical method for fourth order two-point boundary value problems with pantograph type delay are presented.
\end{abstract}

2010 Mathematics Subject Classification: 65D07, 41A15, 65D32.

Key words and phrases: deficient complete quartic spline, error bound, corrected Simpson's quadrature rule.

\section{Introduction}

In this paper we intend to improve the error bound in the interpolation with quartic $\mathrm{C}^{2}$-spline stated in Theorem 3 from [6] in terms of the modulus of continuity for less smooth class of functions which are only continuous. In the $\mathrm{C}^{5}$-smoothness class, several error bounds for complete quartic spline with deficiency 2 were established (see [4], [6], [11], and [14]). This deficient complete quartic spline $s \in S\left(4, \Delta_{n}\right)$, $s:[0, a] \rightarrow \mathbb{R}, s \in C^{2}[0, a]$, is uniquely determined by the interpolation conditions

$$
s\left(x_{i}\right)=f\left(x_{i}\right), i=\overline{0, n}, s\left(\frac{x_{i-1}+x_{i}}{2}\right)=f\left(\frac{x_{i-1}+x_{i}}{2}\right), i=\overline{1, n}
$$

\footnotetext{
${ }^{1}$ Received 1 November, 2019

Accepted for publication (in revised form) 25 November, 2019
} 


$$
s^{\prime}(0)=f^{\prime}(0), \quad s^{\prime}(a)=f^{\prime}(a)
$$

for given values

$$
\begin{gathered}
y_{i}=f\left(x_{i}\right), i=\overline{0, n}, \quad y_{i / 2}=f\left(\frac{x_{i-1}+x_{i}}{2}\right), i=\overline{1, n}, \\
y_{0}^{\prime}=f^{\prime}(0), y_{n}^{\prime}=f^{\prime}(a)
\end{gathered}
$$

on the knots $x_{i}, i=\overline{0, n}$, and on the midpoints of the intervals $\left[x_{i-1}, x_{i}\right], i=\overline{1, n}$, of the partition $\Delta_{n}: 0=x_{0}<x_{1}<\ldots<x_{n-1}<x_{n}=a$. His expression is

$$
\begin{gathered}
s_{i}(x)=\frac{\left(x_{i}-x\right)^{2}\left[\left(x_{i}-x\right)^{2}+4\left(x_{i}-x\right)\left(x-x_{i-1}\right)-5\left(x-x_{i-1}\right)^{2}\right]}{h_{i}^{4}} \cdot y_{i-1}+ \\
+\frac{16\left(x-x_{i-1}\right)^{2}\left(x_{i}-x\right)^{2}}{h_{i}^{4}} \cdot y_{i / 2}+ \\
+\frac{\left(x-x_{i-1}\right)^{2}\left[\left(x-x_{i-1}\right)^{2}+4\left(x_{i}-x\right)\left(x-x_{i-1}\right)-5\left(x_{i}-x\right)^{2}\right]}{h_{i}^{4}} \cdot y_{i}+ \\
+\frac{\left(x_{i}-x\right)\left(x-x_{i-1}\right)\left(x_{i-1}+x_{i}-2 x\right)\left[\left(x_{i}-x\right) \cdot m_{i-1}+\left(x-x_{i-1}\right) \cdot m_{i}\right]}{h_{i}^{3}}
\end{gathered}
$$

for $x \in\left[x_{i-1}, x_{i}\right]$, where $h_{i}=x_{i}-x_{i-1}, i=\overline{1, n}$ and the values $m_{i}=s^{\prime}\left(x_{i}\right)$, $i=\overline{0, n}$, are obtained by the smoothnes condition $s \in C^{2}[0,1]$, as the solution of the three-diagonal linear system

$$
\begin{aligned}
& -\frac{1}{h_{i}} \cdot m_{i-1}+\left(\frac{4}{h_{i}}+\frac{4}{h_{i+1}}\right) \cdot m_{i}-\frac{1}{h_{i+1}} \cdot m_{i+1}=\frac{5}{h_{i}^{2}} \cdot y_{i-1}-\frac{5}{h_{i+1}^{2}} \cdot y_{i+1}+ \\
& \quad+\left(\frac{11}{h_{i}^{2}}-\frac{11}{h_{i+1}^{2}}\right) \cdot y_{i}+\frac{16}{h_{i+1}^{2}} \cdot y_{i+1 / 2}-\frac{16}{h_{i}^{2}} \cdot y_{i / 2}, \quad i=\overline{1, n-1}
\end{aligned}
$$

with the end-point conditions: $m_{0}=f^{\prime}(0), m_{n}=f^{\prime}(a)$. This diagonally dominant system has the matriceal form $A \cdot m=d$ with the condition number $K(A) \leq \frac{5}{3}$.

Concerning the error estimate, for $a=1$, in the case $f \in C^{5}[0,1]$ the following result was obtained:

Theorem 2 (in $[6]$ ): Let $f \in C^{5}[0,1]$. Then we have:

$$
|f(x)-s(x)| \leq \frac{C_{0} h^{5}}{5 !} \cdot \max _{x \in[0,1]}\left|f^{V}(x)\right|, \quad x \in[0,1]
$$

where

$$
\begin{gathered}
C_{0}=\left(\frac{1}{30}+\frac{\sqrt{30}}{3}\right) \cdot \sqrt{\left(\frac{1}{4}-\frac{1}{\sqrt{30}}\right)}=\max _{x \in[0,1]}|c(t)| \\
c(t)=\frac{3 t^{2}(1-2 t)(1-t)^{2}+t(1-t)(1-2 t)}{6} .
\end{gathered}
$$


Also we have

$$
\left|f^{\prime}\left(x_{i}\right)-s^{\prime}\left(x_{i}\right)\right| \leq \frac{h^{4}}{6 !} \cdot \max _{x \in[0,1]}\left|f^{V}(x)\right|, \quad i=\overline{1, n-1} .
$$

Furthermore, $C_{0}$ in (5) cannot be improved for an equally spaced partition. Inequality (6) is also best possible. Also we have

$$
\left|f^{\prime}(x)-s^{\prime}(x)\right| \leq c_{1} \frac{h^{4}}{6 !} \cdot \max _{x \in[0,1]}\left|f^{V}(x)\right| .
$$

The best constant $c_{1}$ for the estimate (7) was established in [14]. Similar error estimates were obtained in [4]. Optimal error bounds for complete quartic splines in the case $f \in C^{5}[0,1]$, by considering

$$
\sup _{f \in C^{5}[0,1]} \frac{\|f-s\|}{\left\|f^{V}\right\| \cdot h^{5}}
$$

were obtained in [15]. In [11], the interpolation conditions (1) are generalized as

$$
s\left(x_{i}\right)=f\left(x_{i}\right), i=\overline{0, n}, \quad s\left(x_{i-1}+\theta h_{i}\right)=f\left(x_{i-1}+\theta h_{i}\right), i=\overline{1, n}
$$

with $\theta \in(0,1)$, obtaining the eqistence and uniqueness of the deficient quartic spline interpolant for $\frac{1}{4} \leq \theta \leq \frac{3}{4}$ and the following error estimate:

Theorem 3.1 (in [11]): If $f \in C^{5}[0,1]$, then

$$
|f(x)-s(x)| \leq K \frac{h^{5}}{5 !} \cdot \max _{x \in[0,1]}\left|f^{V}(x)\right|
$$

where $K=\max _{x \in[0,1], \theta \in\left[\frac{1}{4}, \frac{3}{4}\right]} \frac{1}{6}|6| \theta-t\left|t^{2}(1-t)^{2}+\left(k_{1}(\theta)+k_{2}(\theta)\right)\right| K(t, \theta)||$, and

$$
\left|f^{\prime}\left(x_{i}\right)-s^{\prime}\left(x_{i}\right)\right| \leq \frac{\left(k_{1}(\theta)+k_{2}(\theta)\right) h^{4}}{6 !} \cdot \max _{x \in[0,1]}\left|f^{V}(x)\right|, \quad i=\overline{1, n-1}
$$

which are best possible in the limit $\frac{1}{4} \leq \theta \leq \frac{3}{4}$, with

$$
\begin{gathered}
k_{1}(\theta)=\left[(1-\theta) \theta^{3}(4-3 \theta)+(1-\theta)^{3}\left(3 \theta^{2}+2 \theta+1\right)\right] \\
k_{2}(\theta)=\left[\left(3 \theta^{2}-8 \theta+6\right) \theta^{3}+\theta(1-\theta)^{3}(3 \theta+1)\right] \\
|K(t, \theta)|=t(1-t)|\theta-t|\left[\frac{t}{1-\theta}+\frac{1-t}{\theta}\right] .
\end{gathered}
$$

Also we have

$$
\left|f^{\prime}(x)-s^{\prime}(x)\right| \leq C_{0} \frac{h^{4}}{6 !} \cdot \max _{x \in[0,1]}\left|f^{V}(x)\right| .
$$

In the case $s^{\prime}(0)=s^{\prime}(1)=0$, in [6], without proof, is stated the following estimate in terms of the modulus of continuity: 
Theorem 3 (page 66 in [6]): Let $f \in C[0,1]$. If $\left\{x_{i}\right\}_{i=0}^{k}$ is the partition of equally spaced knots, then for $x_{i-1} \leq x \leq \frac{x_{i-1}+x_{i}}{2}$ and $t=\frac{x-\bar{x}_{i-1}}{h}, i=\overline{1, k}$, we have

$$
|f(x)-s(x)| \leq c_{1}(1-t) \omega(f, h) \leq c_{2} \omega(f, h)
$$

where

$$
\begin{gathered}
c_{1}(t)=1+\frac{13}{3} t-3 t^{2}-\frac{58}{3} t^{3}+16 t^{4} \\
c_{2}=\max \left|c_{1}(t)\right| \approx 1.6572, \quad 0 \leq t \leq \frac{1}{2} .
\end{gathered}
$$

Since the error estimate (8) was established for the first half of the intervals $\left[x_{i-1}, x_{i}\right], i=\overline{1, n}$, in that follows we try to extend this estimate for the entire subinterval $\left[x_{i-1}, x_{i}\right]$ for each $i=\overline{1, n}$, improving the constant $c_{2} \approx 1.6572$, and pointing out the estimate in the case of Lipschitzian functions. As applications of the complete quartic spline (3), we present the corresponding quadrature rule for uniform partitions and investigate the possibility to apply the iterated splines technique (see [2] and [3]) based on complete quartic spline, for the numerical solution of the two-point boundary value problem associated to the beam fourth order differential equation with clamped end-conditions (see [7] and [17]).

\section{Main result}

In the case $s^{\prime}(0)=s^{\prime}(a)=0$ and considering $f \in C[0, a]$, we obtain the following result in terms of the modulus of continuity.

Theorem 1 For $f \in C[0, a]$, the error estimate in the interpolation by the complete quartic spline $s \in C^{2}[0, a]$, satisfying (1) and with the end-point conditions $s^{\prime}(0)=$ $s^{\prime}(a)=0$, is

$$
|f(x)-s(x)| \leq\left[\frac{9317}{8192}+\frac{107+51 \sqrt{17}}{384} \cdot \frac{h^{2}}{\bar{h}^{2}}\right] \cdot \omega\left(f, \frac{h}{2}\right)+\frac{1125}{8192} \cdot \omega(f, h)
$$

for all $x \in\left[x_{i-1}, x_{i}\right], i=\overline{1, n}$, where $h=\max _{i=\overline{1, n}} h_{i}$ and $\bar{h}=\min _{i=\overline{1, n}} h_{i}$. In the case of uniform partition, the estimate is

$$
\begin{array}{r}
\quad|f(x)-s(x)| \leq\left(\frac{9317}{8192}+\frac{107+51 \sqrt{17}}{384}\right) \cdot \omega\left(f, \frac{h}{2}\right)+\frac{1125}{8192} \cdot \omega(f, h) \leq \\
\leq 1.963574902 \cdot \omega\left(f, \frac{h}{2}\right)+0.137329102 \cdot \omega(f, h), \forall x \in\left[x_{i-1}, x_{i}\right], \quad i=\overline{1, n} .
\end{array}
$$

Proof. Denoting $d=\left(d_{1}, \ldots, d_{n-1}\right)$, with

(11) $d_{i}=\frac{5}{h_{i}^{2}} \cdot y_{i-1}-\frac{5}{h_{i+1}^{2}} \cdot y_{i+1}+\left(\frac{11}{h_{i}^{2}}-\frac{11}{h_{i+1}^{2}}\right) \cdot y_{i}+\frac{16}{h_{i+1}^{2}} \cdot y_{i+1 / 2}-\frac{16}{h_{i}^{2}} \cdot y_{i / 2}, i=\overline{1, n-1}$ 
and $m=\left(m_{0}, m_{1}, \ldots, m_{n}\right), m_{0}=m_{n}=0$, by the diagonally dominant system (4) we have $\left\|A^{-1}\right\| \leq \frac{4}{3}$ and $\|m\|=\max \left\{\left|m_{i}\right|: i=\overline{0, n}\right\} \leq\left\|A^{-1}\right\| \cdot \max \left\{\left|d_{i}\right|: i=\overline{1, n-1}\right\}$. Since $y_{i}=f\left(x_{i}\right), i=\overline{0, n}, y_{i / 2}=f\left(\frac{x_{i-1}+x_{i}}{2}\right), i=\overline{1, n}$, by (11) we get

$$
\left|d_{i}\right| \leq \frac{4 h_{i}}{h_{i+1}\left(h_{i}+h_{i+1}\right)} \cdot \omega\left(f, \frac{h_{i+1}}{2}\right)+\frac{4 h_{i+1}}{h_{i}\left(h_{i}+h_{i+1}\right)} \cdot \omega\left(f, \frac{h_{i}}{2}\right), \quad \forall i=\overline{1, n-1}
$$

obtaining,

$$
\left|d_{i}\right| \leq \frac{4 h}{\bar{h}^{2}} \cdot \omega\left(f, \frac{h}{2}\right), \quad \forall i=\overline{1, n-1}
$$

and so, $\|m\| \leq \frac{16 h}{3 \bar{h}^{2}} \cdot \omega\left(f, \frac{h}{2}\right)$. Considering the notation $t=\frac{x-x_{i-1}}{h_{i}}, i=\overline{1, n}$, the expression (3) becomes

$s_{i}(t)=P_{1}(t) \cdot s_{i}(0)+P_{2}(t) \cdot s_{i}\left(\frac{1}{2}\right)+P_{3}(t) \cdot s_{i}(1)+h_{i}\left(P_{4}(t) \cdot s_{i}^{\prime}(0)+P_{5}(t) \cdot s_{i}^{\prime}(1)\right)$

where,

$$
\begin{aligned}
& P_{1}(t)=(1-t)^{2}(1-2 t)(1+4 t) \\
& P_{2}(t)=16 t^{2}(1-t)^{2} \\
& P_{3}(t)=t^{2}(2 t-1)(5-4 t) \quad, \quad t \in[0,1] \\
& P_{4}(t)=t(1-t)^{2}(1-2 t) \\
& P_{5}(t)=t^{2}(1-t)(1-2 t)
\end{aligned}
$$

and consequently,

$$
\begin{gathered}
\max _{x \in\left[x_{i-1}, x_{i}\right]}\left|\frac{\left(x_{i}-x\right)^{2}\left(x_{i-1}+x_{i}-2 x\right)\left(x-x_{i-1}\right)}{h_{i}^{3}}\right|=\left|P_{4}\left(\frac{7-\sqrt{17}}{16}\right)\right| \cdot h_{i}= \\
=\frac{107+51 \sqrt{17}}{4096} \cdot h_{i}
\end{gathered}
$$

and

$$
\begin{gathered}
\max _{x \in\left[x_{i-1}, x_{i}\right]}\left|\frac{\left(x-x_{i-1}\right)^{2}\left(x_{i-1}+x_{i}-2 x\right)\left(x_{i}-x\right)}{h_{i}^{3}}\right|=\left|P_{5}\left(\frac{9+\sqrt{17}}{16}\right)\right| \cdot h_{i}= \\
=\frac{107+51 \sqrt{17}}{4096} \cdot h_{i} .
\end{gathered}
$$

Because for $t \in\left[0, \frac{1}{2}\right]$ we have $P_{1}(t) \geq 0, P_{2}(t) \geq 0, P_{4}(t) \geq 0, P_{5}(t) \geq 0$ and $P_{3}(t) \leq 0$, on the first half interval $\left[x_{i-1}, x_{i-1}+\frac{1}{2}\left(x_{i}-x_{i-1}\right)\right]$ the estimate will be

$$
\begin{gathered}
|f(x)-s(x)| \leq\left|P_{1}(t)+P_{2}(t)\right| \cdot \max \left\{\left|f(x)-y_{i-1}\right|,\left|f(x)-y_{i / 2}\right|\right\}+ \\
+\left|P_{3}(t)\right| \cdot\left|f(x)-y_{i}\right|+\max _{t \in\left[0, \frac{1}{2}\right]}\left|P_{4}(t)\right| h_{i} \cdot\left|m_{i-1}\right|+\max _{t \in\left[0, \frac{1}{2}\right]}\left|P_{5}(t)\right| h_{i} \cdot\left|m_{i}\right| \leq
\end{gathered}
$$




$$
\begin{gathered}
\leq \frac{9317}{8192} \cdot \max \left\{\left|f(x)-y_{i-1}\right|,\left|f(x)-y_{i / 2}\right|\right\}+\frac{1125}{8192} \cdot\left|f(x)-y_{i}\right|+ \\
+\frac{(107+51 \sqrt{17}) h_{i}}{4096} \cdot\left|m_{i-1}\right|+\frac{(107+51 \sqrt{17}) h_{i}}{4096} \cdot\left|m_{i}\right| \leq \frac{9317}{8192} \cdot \omega\left(f, \frac{h}{2}\right)+ \\
+\frac{1125}{8192} \cdot \omega(f, h)+\frac{(107+51 \sqrt{17})}{4096} \cdot \frac{32 h^{2}}{3 \bar{h}^{2}} \cdot \omega\left(f, \frac{h}{2}\right), x \in\left[x_{i-1}, x_{i-1}+\frac{1}{2}\left(x_{i}-x_{i-1}\right)\right] .
\end{gathered}
$$

Similarly, on the second half interval $\left[x_{i-1}+\frac{1}{2}\left(x_{i}-x_{i-1}\right), x_{i}\right]$, for $t \in\left[\frac{1}{2}, 1\right]$, we have $P_{1}(t) \leq 0, P_{4}(t) \leq 0, P_{5}(t) \leq 0$ and $P_{2}(t) \geq 0, P_{3}(t) \geq 0$, and the estimate is

$$
\begin{gathered}
|f(x)-s(x)| \leq\left|P_{2}(t)+P_{3}(t)\right| \cdot \max \left\{\left|f(x)-y_{i}\right|,\left|f(x)-y_{i / 2}\right|\right\}+ \\
+\left|P_{1}(t)\right| \cdot\left|f(x)-y_{i-1}\right|+\max _{t \in\left[\frac{1}{2}, 1\right]}\left|P_{4}(t)\right| h_{i} \cdot\left|m_{i-1}\right|+\max _{t \in\left[\frac{1}{2}, 1\right]}\left|P_{5}(t)\right| h_{i} \cdot\left|m_{i}\right| \leq \\
\leq \frac{1125}{8192} \cdot\left|f(x)-y_{i-1}\right|+\frac{9317}{8192} \cdot \max \left\{\left|f(x)-y_{i}\right|,\left|f(x)-y_{i / 2}\right|\right\}+ \\
+\frac{(107+51 \sqrt{17}) h_{i}}{4096} \cdot\left|m_{i-1}\right|+\frac{(107+51 \sqrt{17}) h_{i}}{4096} \cdot\left|m_{i}\right| \leq \frac{1125}{8192} \cdot \omega(f, h)+ \\
+\frac{9317}{8192} \cdot \omega\left(f, \frac{h}{2}\right)+\frac{(107+51 \sqrt{17})}{4096} \cdot \frac{32 h^{2}}{3 \bar{h}^{2}} \cdot \omega\left(f, \frac{h}{2}\right), x \in\left[x_{i-1}+\frac{1}{2}\left(x_{i}-x_{i-1}\right), x_{i}\right] .
\end{gathered}
$$

Then, we get the estimate

$$
|f(x)-s(x)| \leq\left(\frac{9317}{8192}+\frac{107+51 \sqrt{17}}{384} \cdot \frac{h^{2}}{\bar{h}^{2}}\right) \cdot \omega\left(f, \frac{h}{2}\right)+\frac{1125}{8192} \cdot \omega(f, h)
$$

for all $x \in\left[x_{i-1}, x_{i}\right], i=\overline{1, n}$. In the case of uniform partition this inequality becomes

$$
|f(x)-s(x)| \leq\left(\frac{9317}{8192}+\frac{107+51 \sqrt{17}}{384}\right) \cdot \omega\left(f, \frac{h}{2}\right)+\frac{1125}{8192} \cdot \omega(f, h)
$$

obtaining (10).

Corollary 1 If the function $f$ is Lipschitzian, $f \in \operatorname{Lip}_{L}[0, a]$, in the case of uniform partition the error estimate (10) becomes

$$
\begin{aligned}
& |f(x)-s(x)| \leq\left(\frac{11567}{16384}+\frac{107+51 \sqrt{17}}{768}\right) \cdot L h \leq \\
& \leq 1.119117 \cdot L h, \quad \forall x \in\left[x_{i-1}, x_{i}\right], \quad i=\overline{1, n} .
\end{aligned}
$$

Remark 1 If $f \in \operatorname{Lip}_{L}[0,1]$, then the error estimate (8) from [6] becomes

$$
|f(x)-s(x)| \leq 1.6572 \cdot L h, \quad \forall x \in\left[x_{i-1}, \frac{x_{i-1}+x_{i}}{2}\right], i=\overline{1, n}
$$

and we see that the estimate (12) is better. 


\section{Applications of complete quartic spline}

\subsection{Numerical integration}

Integrating on $[0, a]$ the interpolation formula provided by the complete quartic spline (3) on equally spaced knots, with given values of the derivative on the endpoints $f^{\prime}(0), f^{\prime}(a)$, we obtain the following quadrature rule

$$
\begin{gathered}
\int_{0}^{a} f(x) d x=\frac{a}{30 n} \cdot \sum_{i=1}^{n}\left[7 f\left(x_{i-1}\right)+16 f\left(\frac{x_{i-1}+x_{i}}{2}\right)+7 f\left(x_{i}\right)\right]- \\
-\frac{a^{2}}{60 n^{2}} \cdot\left[f^{\prime}(a)-f^{\prime}(0)\right]+R_{n}(f)
\end{gathered}
$$

which is just the corrected Simpson's quadrature rule with the error estimate in the case $f \in C^{5}[0, a]$,

$$
\left|R_{n}(f)\right| \leq \frac{a^{6}}{23040 n^{5}} \cdot\left\|f^{V}\right\|_{\infty} .
$$

Usually, the corrected Simpson's quadrature rule is obtained by the classical way of Richardson extrapolation. Here, it was obtained as an interpolatory quadrature rule generated by the quartic Hermite interpolation polynomial given by the conditions on $f(0), f\left(\frac{a}{2}\right), f(a), f^{\prime}(0), f^{\prime}(a)$. Another way to obtain the same corrected Simpson's quadrature rule is the use of finite differences (see [12]). The quadrature rule (13) was generalized in [8] as follows:

$$
\begin{gathered}
\int_{0}^{a} f(x) d x=\frac{a}{n} \cdot \sum_{i=1}^{n}\left[\frac{\theta}{2}\left(f\left(x_{i-1}\right)+7 f\left(x_{i}\right)\right)+(1-\theta) f\left(\frac{x_{i-1}+x_{i}}{2}\right)\right]- \\
-\frac{(1-3 \theta) a^{2}}{24 n^{2}} \cdot\left[f^{\prime}(a)-f^{\prime}(0)\right]+R_{n}(f), \quad \theta \in[0,1]
\end{gathered}
$$

here, formula (13) being obtained for $\theta=\frac{7}{15}$. Another generalization based on the extended Euler formula was obtained in [10], and an extended quadrature formula for functions with absolutely continuous derivative $f^{(m-1)}$ was obtained in [13]. An extension of the quadrature formula (13) with variable weights was proposed in [5]. Using the Bernoulli polynomials, the study of corrected trapezoidal, midpoint, and Simpson's rule was unified in [9], provinding the corresponding error estimates.

As we can see, the order of convergence of the quadrature rule (13)-(14) is $O\left(h^{5}\right)$ and it is expected to provide better results than the Simpson's rule which has the order $O\left(h^{4}\right)$. Another quadrature rule of order $O\left(h^{4}\right)$ is the corrected trapezoidal rule investigated in [1]. For testing the performances of the quadrature rule (13)-(14) in comparison with the Simpson's rule and with the corrected trapezoidal rule we consider the following two examples

$$
\pi=\text { displaystyle } \int_{0}^{1} \frac{4}{1+x^{2}} d x \text { and } \operatorname{erf}(1)=\frac{2}{\sqrt{\pi}} \int_{0}^{1} e^{-x^{2}} d x
$$


and applying the corrected Simpson's rule (CSR), the Simpson's rule (SR), and the corrected trapezoidal rule (CTR) for stepsize $h=0.1$ and $h=0.05$ we obtain the following results.

\begin{tabular}{|l|l|l|}
\hline$\pi$ & error, $\mathrm{n}=10$ & error, $\mathrm{n}=20$ \\
\hline TCR & $1.984 \times 10^{-9}$ & $3.1002 \times 10^{-11}$ \\
\hline SR & $6.2001 \times 10^{-10}$ & $9.6878 \times 10^{-12}$ \\
\hline CSR & $9.9200 \times 10^{-11}$ & $1.5494 \times 10^{-12}$ \\
\hline$e r f(1)$ & error, $\mathrm{n}=10$ & error, $\mathrm{n}=20$ \\
\hline TCR & $2.305 \times 10^{-7}$ & $1.4412 \times 10^{-8}$ \\
\hline SR & $5.7619 \times 10^{-8}$ & $3.6025 \times 10^{-9}$ \\
\hline CSR & $5.8760 \times 10^{-12}$ & $3.7137 \times 10^{-13}$ \\
\hline
\end{tabular}

Table 1. The accuracy of the rule (13)

As can be observed, the best results were obtained with the corrected Simpson's rule and for the case of error-function value $\operatorname{erf}(1)$, this is highly significant.

\subsection{Iterative numerical method for two-point boundary value prob- lems}

The fourth order boundary value problem modelling the bending of a rectangular clamped beam of length $L$ resting on an elastic foundation (see [7]) has the form:

$$
\left\{\begin{array}{c}
x^{I V}(t)=f(t, x(t)), \quad t \in[0, L] \\
x(0)=c, \quad x(L)=d, \quad x^{\prime}(0)=w, \quad x^{\prime}(L)=r
\end{array}\right.
$$

and generalizes the Euler's model

$$
\left\{\begin{array}{c}
x^{I V}(t)+\frac{K}{D} \cdot x(t)=\frac{1}{D} \cdot f(t), \quad t \in[0, L] \\
x(0)=0, \quad x(L)=0, \quad x^{\prime}(0)=0, \quad x^{\prime}(L)=0
\end{array} .\right.
$$

Since recently was investigated the second order two-point boundary value problem with pantograph type delay (see [16])

$$
\left\{\begin{array}{c}
x^{\prime \prime}(t)=f(t, x(t), x(q t)), \quad t \in[0, a], q \in(0,1) \\
x(0)=c, \quad x(a)=d
\end{array}\right.
$$

we can consider the generalization of (15)

$$
\left\{\begin{array}{c}
x^{I V}(t)=f(t, x(t), x(q t)), \quad t \in[0, a] \\
x(0)=c, \quad x(a)=d, \quad x^{\prime}(0)=w, \quad x^{\prime}(a)=r .
\end{array}\right.
$$

The equivalent integral form of (16) is

$$
x(t)=g(t)+\int_{0}^{a} G(t, s) \cdot f(s, x(s), x(q s)) d s, \quad t \in[0, a]
$$

with

$$
g(t)=\frac{(a-t)^{2}(2 t+a) \cdot c+t^{2}[2(a-t)+a] \cdot d}{a^{3}}+
$$




$$
+\frac{t(a-t)^{2} \cdot w-t^{2}(a-t) \cdot r}{a^{2}}, \quad t \in[0, a]
$$

and the Green function

$$
G(t, s)=\left\{\begin{array}{l}
\frac{1}{6}\left(\frac{s}{a}\right)^{2}\left(1-\frac{t}{a}\right)^{2} \cdot\left[\frac{t-s}{a}+2\left(1-\frac{s}{a}\right)\left(\frac{t}{a}\right)\right], s \leq t \\
\frac{1}{6}\left(\frac{t}{a}\right)^{2}\left(1-\frac{s}{a}\right)^{2} \cdot\left[\frac{s-t}{a}+2\left(1-\frac{t}{a}\right)\left(\frac{s}{a}\right)\right], s \geq t
\end{array} .\right.
$$

Considering the Lipschitz constants of $f$ with respect to the second and to the third argument be $\alpha$ and $\beta$, the existence and uniqueness of the solution of (16) is ensured by the contraction condition $\frac{a(\alpha+\beta)}{192}<1$. By using the Picard iterations associated to the integral equation (17), we can develop the technique of iterated splines (see [2] and [3]) providing an iterative algorithm for solving (17) on the equally spaced knots $t_{i}=\frac{i \cdot a}{n}, \quad i=\overline{0, n}$,

$$
\begin{gathered}
x_{k}\left(t_{i-1}+\gamma_{m} \cdot h\right)=g\left(t_{i-1}+\gamma_{m} \cdot h\right)+\frac{a}{2 n} \cdot \sum_{j=1}^{n}\left[G\left(t_{i-1}+\gamma_{m} \cdot h, t_{j-1}\right) .\right. \\
\cdot f\left(t_{j-1}, x_{k-1}\left(t_{j-1}\right), s_{k-1}\left(q \cdot t_{j-1}\right)\right)+G\left(t_{i-1}+\gamma_{m} \cdot h, t_{j}\right) . \\
\left.f\left(t_{j}, x_{k-1}\left(t_{j}\right), s_{k-1}\left(q \cdot t_{j}\right)\right)\right], \quad i=\overline{1, n}, \gamma_{0}=0, \gamma_{1}=\frac{1}{2}, \gamma_{2}=1, k \in \mathbb{N}^{*}
\end{gathered}
$$

where $h=\frac{a}{n}$ and $x_{0}(t)=g(t)$.

Here, $s_{k-1}$ is the complete quartic spline interpolating the values $x_{k-1}\left(t_{j}\right), j=$ $\overline{0, n}, x_{k-1}\left(\frac{t_{i-1}+t_{i}}{2}\right), i=\overline{1, n}$ and with the end-point conditions

$$
s_{k-1}^{\prime}(0)=w, \quad s_{k-1}^{\prime}(a)=r .
$$

The stopping criterion is: $\left|x_{k}\left(t_{i}\right)-x_{k-1}\left(t_{i}\right)\right|<\varepsilon, \forall i=\overline{1, n-1}$, and the iterative sequence $\left(x_{k}\left(t_{i}\right)\right)_{k \in \mathbb{N}}$ approximates the exact solution $x^{*}\left(t_{i}\right)$ on the knots $t_{i}, i=\overline{1, n-1}$. Concerning the error estimate of the method, in the case $f \in$ $C^{5}([0, a] \times \mathbb{R} \times \mathbb{R})$, if $\frac{a\left(c_{1} \alpha+c_{2} \beta\right)}{192}<1$, then the following inequality is obtained:

$$
\begin{gathered}
\left|x_{k}\left(t_{i}\right)-x^{*}\left(t_{i}\right)\right| \leq \frac{\left(\frac{a(\alpha+\beta)}{192}\right)^{m}}{1-\frac{a(\alpha+\beta)}{192}} \cdot \frac{a M_{0}}{192}+ \\
+\frac{a M_{4} \cdot h^{4}}{720\left[1-\frac{a\left(c_{1} \alpha+c_{2} \beta\right)}{192}\right]}+\frac{c\left\|x_{k-1}^{V}\right\|_{\infty} \cdot h^{5}}{1-\frac{a(\alpha+\beta)}{192}}, \quad i=\overline{1, n-1, k \in \mathbb{N}^{*}}
\end{gathered}
$$

where $M_{0}=\max \{|f(s, g(s), g(q s))|: s \in[0, a]\}, M_{4}=\left\|F_{k, i}^{I V}\right\|_{\infty}$,

$$
F_{k, i}^{I V}\left(t_{i-1}+\gamma_{m} \cdot h, s\right)=G\left(t_{i-1}+\gamma_{m} \cdot h, s\right) \cdot f\left(s, x_{k}(s), x_{k}(q s)\right), m=\overline{0,2}
$$

$k \in \mathbb{N}^{*}, i=\overline{1, n}, \gamma_{0}=0, \gamma_{1}=\frac{1}{2}, \gamma_{2}=1$, and $c_{1}, c_{2}>1, c>0$ are suitable constants. The order of convergence is $O\left(h^{4}\right)$, but having a term with the order $O\left(h^{5}\right)$. 
According to [2] and [3], the error estimate of the method which use the complete cubic spline as interpolation procedure is the same $O\left(h^{4}\right)$ and the expression of the complete cubic spline at each iterative step is more simple

$s_{k-1}^{(i)}(t)=\frac{\left(t_{i}-t\right)^{2}\left[2\left(t-t_{i-1}\right)+h\right]}{h^{3}} \cdot x_{k-1}\left(t_{i-1}\right)+\frac{\left(t-t_{i-1}\right)^{2}\left[2\left(t_{i}-t\right)+h\right]}{h^{3}} \cdot x_{k-1}\left(t_{i}\right)+$

$+\frac{\left(t_{i}-t\right)^{2}\left(t-t_{i-1}\right)}{h^{2}} \cdot m_{k-1}^{(i-1)}+\left(-\frac{\left(t-t_{i-1}\right)^{2}\left(t_{i}-t\right)}{h^{2}}\right) \cdot m_{k-1}^{(i)}, \quad \forall t \in\left[t_{i-1}, t_{i}\right], i=\overline{1, n}$

where $m_{k-1}^{(0)}=w, m_{k-1}^{(n)}=r$, and $m_{k-1}^{(i)}, i=\overline{1, n-1}$, represent the solution of the tridiadonal, diagonally dominant, linear system:

$$
m_{k-1}^{(i-1)}+4 m_{k-1}^{(i)}+m_{k-1}^{(i+1)}=\frac{3\left(x_{k-1}\left(t_{i+1}\right)-x_{k-1}\left(t_{i-1}\right)\right)}{h}, \quad i=\overline{1, n-1} .
$$

For comparing the results provided by the iterated splines method which uses the interpolation procedures of complete cubic splines and complete quartic splines, rspectively, we consider the following numerical experiment:

The two-point boundary value problem

$$
\left\{\begin{array}{c}
x^{I V}(t)=\frac{22}{(t+1)^{5}}+\frac{1}{(t+1)^{2}} \cdot\left([x(t)]^{2}+[x(t)]^{3}\right) x\left(\frac{t}{2}\right), \quad t \in[0,1] \\
x(0)=1, \quad x(1)=0.5, \quad x^{\prime}(0)=-1, \quad x^{\prime}(1)=-0.25
\end{array}\right.
$$

has the exact solution $x^{*}(t)=\frac{1}{t+1}$, and applying the iterative algorithm (18) for $n=5$ and $n=25$, with $\varepsilon=10^{-15}$, the number of iterations will be $k=7$. The numerical results are presented in Table 2 , where the absolute errors on the knots are $e_{i}=\left|x^{*}\left(t_{i}\right)-x_{k}\left(t_{i}\right)\right|, i=\overline{0, n}$. The involved quadrature rule is the same corrected trapezoidal rule for the algorithms (18) and (19).

\begin{tabular}{|l|l|l|}
\hline$t_{i}$ & $e_{i}, \mathrm{n}=5$ & $e_{i}, \mathrm{n}=25$ \\
\hline 0 & 0 & 0 \\
\hline 0.2 & $1.504103 \mathrm{e}-04$ & $7.657559 \mathrm{e}-06$ \\
\hline 0.4 & $1.893587 \mathrm{e}-04$ & $1.434412 \mathrm{e}-05$ \\
\hline 0.6 & $1.367446 \mathrm{e}-04$ & $1.233097 \mathrm{e}-05$ \\
\hline 0.8 & $4.697401 \mathrm{e}-05$ & $4.805814 \mathrm{e}-06$ \\
\hline 1 & 0 & 0 \\
\hline
\end{tabular}

Table 2. Results with algorithm (18)

With the iterative algorithm (19), for $n=10$ and $n=100$, with $\varepsilon=10^{-15}$, the number of iterations is again $k=7$ and the numerical results are presented in Table 3 . 


\begin{tabular}{|l|l|l|}
\hline$t_{i}$ & $e_{i}, \mathrm{n}=10$ & $e_{i}, \mathrm{n}=100$ \\
\hline 0 & 0 & 0 \\
\hline 0.2 & $7.702524 \mathrm{e}-06$ & $8.044273 \mathrm{e}-10$ \\
\hline 0.4 & $8.374001 \mathrm{e}-06$ & $8.727925 \mathrm{e}-10$ \\
\hline 0.6 & $5.409719 \mathrm{e}-06$ & $5.634118 \mathrm{e}-10$ \\
\hline 0.8 & $1.701300 \mathrm{e}-06$ & $1.772970 \mathrm{e}-10$ \\
\hline 1 & 0 & 0 \\
\hline
\end{tabular}

Table 3. Results with algorithm (19)

We see that the order of convergence in Table 2 seems to be weaker than $O\left(h^{4}\right)$, while in Table 3 the order $O\left(h^{4}\right)$ is confirmed. So we can say that better results are obtained by using cubic splines.

\section{Conclusions}

In the case of continuous functions $f \in C[0,1]$, the error bound obtained in Theorem 1 is an improvement of the corresponding results Theorem 3 from [6] providing a smaller constant. This fact becomes obvious in Corollary 1 for the case of Lipschitzian functions. For the cases $f \in C^{1}[0,1]$ and $f \in C^{2}[0,1]$, corresponding error estimates could be obtained by using the first and the second modulus of smoothness, respectively. Concerning the quadrature rule generated by the complete quartic spline interpolation, superior performances in numerical integration are guaranted in comparison with the Simpson's rule. The advantage is more clear considering the computation of only two additional terms in formula (13) for any stepsize $\frac{a}{n}$ (not depending by $n$ ), given in the expression $\frac{a^{2}}{60 n^{2}} \cdot\left[f^{\prime}(a)-f^{\prime}(0)\right]$, but the accuracy is increased by $O\left(h^{4}\right)$ to $O\left(h^{5}\right)$. These are confirmed investigating the results from Table 1. The situation is not the same for complicated algorithms. Despite of the fact that theoretically the order of error should be better by using quartic splines than those involving cubic splines, the numerical results after implementation contradict this expectation as can be viewed in Tables 2 and 3. The reason is in the accumulation of errors for highly elaborated algorithms by a step to another. Considering this fact of complexity of calculus, the use of elaborated algorithms is not recommendable, the simpler algorithm being more useful in practice. This is the situation for the alternative of choosing the interpolation procedure in the application of the iterated splines method for the boundary value problem (16), the use of the simpler cubic spline being more effective.

Acknowledgement: The first author dedicates this work to the memory of Professor Crăciun Iancu (1939-2007) for his generous support during the first period of formation as researcher and for introducing in the research field of splines.

The authors are thankful to Professor Florin Sofonea and to Professor Ioan Ţincu for the fruitful discussions about the complexity of calculus. 


\section{References}

[1] N. S. Barnett, S.S. Dragomir, A perturbed trapezoid inequality in terms of the fourth derivative, Korean J. Comput. Appl. Math., vol. 9, no. 1, 2002, 45-60.

[2] A. M. Bica, M. Curilă, S. Curilă, Two-point boundary value problems associated to functional differential equations of even order solved by iterated splines, Appl. Numer. Math., vol. 110, 2016, 128-147.

[3] A. M. Bica, M. Curilă, S. Curilă, Spline iterative method for pantograph type functional differential equations, in Finite Difference Methods. Theory and Applications, I. Dimov, I. Farago, L. Vulkov (eds.), LNCS 11386, Springer Nature Switzerland, 2019, 159-166.

[4] S. Dubey, Y. P. Dubey, Convergence of $C^{2}$ deficient quartic spline interpolation, Adv. Comput. Sciences \& Technol., vol. 10, no. 4, 2017, 519-527.

[5] I. Franjić, J. Pečarić, I. Perić, General three-point quadrature formulas of Euler type, ANZIAM J., vol. 52, 2011, 309-317.

[6] G. Howell, A. K. Varma, Best error bounds for quartic spline interpolation, J. Approx. Theory, vol. 58, 1989, 58-67.

[7] P. Korman, Computation of displacements for nonlinear elastic beam models using monotone iterations, Int. J. Math. Math. Sci., vol. 11, 1988, 121-128.

[8] Z. Liu, New sharp error bounds for some corrected quadrature formulae, Sarajevo J. Math., vol. 9, no. 21, 2013, 37-45.

[9] Z. Liu, Error estimates for some composite corrected quadrature rules, Appl. Math. Letters, vol. 22, 2009, 771-775.

[10] J. Pečarić, I. Franjić, Generalization of corrected Simpson's formula, ANZIAM J., vol. 47, 2006, 367-385.

[11] S.S. Rana, Y. P. Dubey, Best error bounds for decient quartic spline interpolation, Indian J. Pure Appl. Math., vol. 30, no. 4, 1999, 385-393.

[12] N. Ujević, A. J. Roberts, A corrected quadrature formula and applications, ANZIAM J., vol. 45, 2004, 41-56.

[13] N. Ujević, A generalization of the modified Simpson's rule and error bounds, ANZIAM J., vol. 47, 2005, E1-E13.

[14] Yu. S. Volkov, Best error bounds for the derivative of a quartic interpolation spline, Mat. Trud., vol. 1, no. 2, 1998, 68-78 (in Russian).

[15] Wang Jianzhong, Huang Daren, On quartic and quintic interpolation splines and their optimal error bounds, Scientia Sinica (Ser. A), vol. 25, no. 11, 1982, 1130-1141. 
[16] A.-M. Wazwaz, M. A. Z. Raja, M. I. Syam, Reliable treatment for solving boundary value problems of pantograph delay differential equation, Rom. Rep. Phys., vol. 69 , art. 102, 2017.

[17] B. Yang, Estimates of positive solutions to a boundary value problem for the beam equation, Commun. Math. Anal., vol. 2, no. 1, 2007, 13-21.

\author{
Alexandru Mihai Bica \\ University of Oradea \\ Faculty of Informatics and Sciences \\ Department of Mathematics and Informatics \\ Address: Universităţii no. 1, 410087, Oradea, Romania \\ e-mail: abica@uoradea.ro
}

\title{
Diana Curilă
}

University of Oradea

Faculty of Informatics and Sciences

Department of Mathematics and Informatics

Address: Universităţii no. 1, 410087, Oradea, Romania

e-mail: curila_diana@yahoo.com

\section{Zoltan Satmari}

University of Oradea

Faculty of Informatics and Sciences

Department of Mathematics and Informatics

Address: Universităţii no. 1, 410087, Oradea, Romania

e-mail: zsatmari@yahoo.com 